Archives

40 | 2007

Dépendance(s)

\title{
Le travail, l'usine, la chaîne
}

Une trop belle évidence?

\section{Nicolas Hatzfeld}

\section{(2) OpenEdition}

Journals

Édition électronique

URL : http://journals.openedition.org/ccrh/3376

DOI : $10.4000 /$ ccrh.3376

ISSN : $1760-7906$

Éditeur

Centre de recherches historiques - EHESS

Édition imprimée

Date de publication : 25 avril 2007

Pagination : 127-139

ISSN : 0990-9141

Référence électronique

Nicolas Hatzfeld, «Le travail, l'usine, la chaîne », Les Cahiers du Centre de Recherches Historiques [En ligne], 40 | 2007, mis en ligne le 11 octobre 2011, consulté le 10 décembre 2020. URL : http:// journals.openedition.org/ccrh/3376 ; DOI : https://doi.org/10.4000/ccrh.3376

Ce document a été généré automatiquement le 10 décembre 2020.

Article L.111-1 du Code de la propriété intellectuelle. 


\title{
Le travail, l'usine, la chaîne
}

\author{
Une trop belle évidence?
}

\author{
Nicolas Hatzfeld
}

\section{Introduction}

1 Parmi les situations de dépendance dans les sociétés contemporaines figure la situation des ouvriers d'usine. Leur condition hérite de l'image du prolétaire, apparue au temps des manufactures et consolidée par les écrits socialistes. En évoluant, le monde des ouvriers transforme l'image de dépendance. L'usine concrétise à travers ses machines et ses installations autant qu'avec ses règles le poids de la technique et de l'autorité, tandis que les liens tissés par l'entreprise avec son personnel se recomposent ${ }^{1}$. Dans ce monde, le travail à la chaîne fonctionne comme un idéal-type du travail sous contrainte, tandis que les concentrations industrielles rassemblent avec une force peu comparable l'hégémonie d'une entreprise sur un territoire et sur la société qui y vit. Ces formes de domination renvoient à deux notions phares de l'histoire sociale, et plus largement des sciences sociales appliquées à la société industrielle, le taylorisme et le paternalisme. Les deux termes pèsent parfois au point de surdéterminer les débats. Ainsi certains retrouvent, dans les réalités qu'ils observent, la confirmation de ces notions tandis que d'autres, au contraire, appellent à en finir avec elles. Or, précisément, la fortune de ces notions est à rapporter à l'enjeu de la dépendance. Le taylorisme est souvent pensé comme la prise de contrôle du travail ouvrier et plus largement des ateliers par l'encadrement et ses bureaux de techniciens. Le paternalisme désigne selon André Gueslin un "système régissant les relations entre employeurs et salariés d'une entreprise dans leur totalité $»^{2}$.

2 Puissante concentration industrielle, le monde sochalien de Peugeot est un bon ancrage pour éprouver l'idée de dépendance, la réinterroger sans partir des notions phares mais en s'appuyant sur l'étude des pratiques. On peut, pour cela, se référer à deux termes couramment usités par les ouvriers pour évoquer leur travail, ceux de poste et de place. Tandis que le second désigne l'emploi, le premier caractérise l'affectation précise et l'activité qui lui correspond. Ces mots recoupent, grosso modo, les espaces de référence correspondant aux notions évoquées plus haut. Mais les choses suivent ici le point de vue 
des salariés qui distinguent ces références sans les séparer totalement. L'époque examinée, enfin, est essentiellement la seconde moitié du XXe siècle : l'histoire a à faire entendre sa voix à propos de ce temps proche, occupé par les sciences sociales conjuguées au présent.

\section{Du côté de l'emploi : des rémunérations avantageuses et assurées}

3 Couramment usitée dans la région, l'expression « une place chez Peugeot » met l'accent sur le fait d'être admis dans la maison, d'en faire partie et d'être ainsi pris dans l'ensemble des liens que l'entreprise tisse dans la région. Cet ensemble est ainsi présenté au début des années quatre-vingt :

De la colline de Grand Charmont, au-dessus de Sochaux, on domine l'univers mécanique placé sous le signe du lion jaune et bleu. Couleurs, image et lettres qui emplissent le paysage, obsèdent le regard Peugeot, tout est Peugeot, le métal et la pierre, la brique et la peau, le souffle et le cri. Peugeotville, Peugeotland, Peugeot tout ${ }^{3}$.

4 La tirade s'appuie sur l'emprise paysagère du centre de production de Sochaux pour affirmer la toute puissance de l'entreprise sur le Pays de Montbéliard.

Parmi les atouts de la firme au lion figure la rémunération des salariés, supérieure à la moyenne régionale. L'écart varie selon les périodes. Au cours des années soixante, il est souvent du simple au double, à qualification égale ou se traduit par le fait qu'un ouvrier de chez Peugeot gagne à peu près autant qu'un contremaître de l'une des petites usines proches, telle que celles du textile vosgien en difficulté. Mais cet avantage n'a pas toujours existé. Dans les années de la reconstruction, les difficultés de recrutement conduisent les dirigeants de l'usine à constater que les salaires de leurs ouvriers sont inférieurs à ceux des entreprises métallurgiques de la région, dont certains de leurs soustraitants. C'est pour remédier à la pénurie de candidatures qu'ils convainquent la direction générale d'entamer un accroissement relatif des salaires. L'écart qui en résulte se maintient durant la période où l'usine embauche massivement, tant pour assurer sa croissance que pour faire face à l'important « turn-over » qui caractérise ces années. Puis, à partir des années quatre-vingt, les ouvriers évoquent avec amertume le tassement de leurs avantages, tandis que les besoins de recrutement se font maigres, et occasionnels.

Le salaire n'est pas seul, mais accompagné d'un système de primes composé dans les années cinquante suivant plusieurs critères entrecroisés : l'activité personnelle du salarié (rendement et assiduité) rythmée par quinzaine et par an, la production de l'usine (régularité de production, bonne marche industrielle, lancement d'un nouveau modèle, suggestions d'amélioration), et la prospérité de l'entreprise (intéressement aux résultats, ventes, bénéfices réinvestis) $)^{4}$. Le salaire n'est pas brut. Des mesures de protection de la rémunération sont mises en place, par étapes. C'est d'abord une assurance contre le chômage, dans le cadre des accords d'entreprise réalisés au cours de la seconde moitié des années cinquante. Les années d'après-1968 voient se consolider le dispositif. En 1970, un accord de mensualisation procure aux ouvriers une stabilisation des revenus supérieure à celle de l'accord national, et présentée par l'entreprise comme la mise en place d'un «statut de l'ouvrier mensuel »5. L'expression renvoie à une aspiration profonde dans le monde ouvrier, illustrée par les créations du statut du mineur ${ }^{6}$ et de l'électricien de 1946 ou du docker en $1947^{7}$. L'aspect «statutaire » est accentué en 1976 quand, à la suite de 
l'accord national sur les classifications signé par l'Union des industries métallurgiques et minières (UIMM) et plusieurs organisations syndicales ${ }^{8}$ en 1975, une grille unique de classification s'applique aux ouvriers comme aux employés, techniciens et agents de maîtrise.

L'accord sur l'Assurance contre les aléas de carrière (ACAC), signé le 8 novembre 1973, décline également le thème de la garantie de rémunération. Dans plusieurs usines automobiles l'époque voit se multiplier des grèves d'ouvriers spécialisés (OS) réclamant notamment la garantie de salaire en cas de mutation ${ }^{9}$, en particulier lorsqu'ils quittent des postes particulièrement pénibles d'ateliers de mécanique, de presses ou de peinture ${ }^{10}$. Ces conflits s'attaquent au système de salaire au poste, aux inégalités, à l'insécurité et à l'absence de perspectives de progression qui lui sont liées. Chez Renault ils trouvent une réponse partielle dans la création de l'échelon de Professionnel de fabrication (P1F) puis son extension. À Sochaux, l'accord sur l'ACAC ne garantit pas expressément le maintien des taux en cas de mutation, mais évite toute perte immédiate de salaire et organise un glissement progressif vers le taux (de salaire et de prime) correspondant à la nouvelle affectation. Le nouveau système, qui amende le salaire au poste sans le supprimer, amorce une individualisation de la rémunération, qui s'engage dans la seconde moitié des années soixante-dix, à la suite de l'accord national sur la classification signé en $1975^{11}$. Celui-ci simplifie la grille des classifications, réduit le poids des qualifications, et dissocie en grande partie les salaires des postes occupés pour les lier à la notion de compétence (connaissances requises, autonomie, responsabilités et le type d'activité exercée). À la satisfaction des réclamations ouvrières s'ajoutent des intérêts managériaux. Les responsables d'usine veulent développer l'adaptabilité des hommes, la mobilité interne et les reconversions du personnel. Dans l'entreprise, l'individualisation ${ }^{12}$ des rémunérations ouvrières est accentuée en 1978 avec la mise en place d'un dossier individuel du personnel ouvrier. Celui-ci étend à cette catégorie le mode de gestion des employés, techniciens et agents de maîtrise, et permet de présenter la gestion de l'ensemble des salariés, des cadres jusqu'aux ouvriers, comme réunies dans une même logique personnalisée. Mais les syndicats CFDT et CGT dénoncent le nouveau système comme un moyen de division et de manipulation des ouvriers, à travers le dossier individuel, l'entretien d'évaluation et les augmentations individuelles. De tels éléments visent, selon eux, à accentuer une forme d'effritement de l'identité collective ouvrière, et y réussissent en bonne partie. Ainsi, dans les groupes, le nombre des ouvriers - militants syndicaux en général - qui continuent à montrer leur fiche de paie se réduit ${ }^{13}$. Toutefois il convient de nuancer cette évolution: les limites drastiques imposées à l'enveloppe globale des salaires restreignent considérablement le jeu managérial et l'impact de cette politique de distinction individualisée.

Par-delà les modifications de règles et de montants de la rémunération, la sécurité de l'emploi constitue un lien implicite. Sans jamais l'afficher, l'entreprise évite en effet que ses réductions d'effectifs se traduisent par des licenciements, ce qui revient à attribuer une garantie d'emploi à ses salariés en titre. L'argument pèse lourd à partir des années quatre-vingt et compense aisément l'affaiblissement de l'avantage salarial antérieur. 


\section{L'éventail des liens d'entreprise : une composition évolutive}

Autour de la rémunération proprement dite, le statut de salarié Peugeot offre un ensemble d'avantages que l'entreprise procure à ses salariés : logement, transports, école d'apprentissage faisant une place particulière aux enfants du personnel, magasins à bas prix, vente de voitures à tarif préférenciel, etc. Là aussi, le tableau évolue, au gré des différences de dynamiques qui affectent ces différents liens noués entre les salariés et l'entreprise.

10 Parmi ces avantages d'entreprise, plusieurs, anciens, connaissent des fortunes diverses qui toutes tendent à éparpiller le lien salarial. Les œuvres sociales ont longtemps participé d'une logique paternaliste teintée de protestantisme ${ }^{14} .1936$ donne lieu à une technicisation de la gestion des questions sociales, qu'illustre la création de techniciens sociaux après la grève $\mathrm{e}^{15}$. Cette évolution est accentuée à la Libération et refondue dans une perspective paritaire dans le cadre du Comité d'entreprise (le CE), créé par l'ordonnance du 22 février 1945. Le lien à l'entreprise se nourrit du développement des activités et services tout en se distinguant d'une stricte logique d'allégeance. Il évolue au gré des majorités syndicales dans sa tonalité comme dans la forme concrète des actions menées. Durant les premières décennies marquées par une hégémonie de la CGT, l'accent est mis sur des activités collectives aussi bien que sur une dimension culturelle critique voire anti-patronale. Le CE forme un contre-pouvoir intérieur à l'entreprise, tandis qu'à l'extérieur, d'autres camarades - parfois les mêmes - mènent un combat politique de gauche. L'éviction de la CGT et l'avènement au début des années quatre-vingt d'une majorité dite participative par la direction de l'entreprise entraîne une réorientation du CE. Celui-ci réduit les activités pratiquées entre "gens» de chez Peugeot ainsi que ses initiatives propres dans le domaine culturel, et développe, sous forme de réductions tarifaires, une dispersion des offres de loisirs dans des organismes extérieurs.

11 D'autres évolutions marquent la fin d'une forme de relation. C'est le cas des magasins Ravi , créés au début de la Première guerre mondiale afin d'assurer le ravitaillement du personnel et maintenu en temps de paix. Après la reconstruction, ces magasins pèsent sur les prix pratiqués par le commerce local, et servent de référence (avec l'indice Insee et face à l'indice CGT) de prix qui étayent les négociations salariales ${ }^{16}$. Des succursales ouvrent dans la plupart des communes du Pays de Montbéliard. Progressivement, le rôle des Ravi évolue. Au fur et à mesure que l'aisance des salariés croît, les magasins offrent des produits d'équipement domestique et d'habillement, accompagnant l'essor de la consommation parmi le personnel. Au début des années quatre-vingt, l'entreprise revend sa chaîne de magasins à une chaîne d'hypermarchés, et prend ainsi acte de l'inutilité de ses pressions sur les prix du commerce, cette fonction étant désormais assurée par la grande distribution devenue hégémonique.

12 Une chronologie voisine marque l'École d'apprentissage Peugeot, créée en $1930^{17}$. Celle-ci doit avant tout fournir l'usine en professionnels d'excellence pour faire face à la crise de l'apprentissage et fidéliser un réseau ouvrier. Les enfants du personnel sont parfois clairement désignés comme candidats privilégiés, tandis qu'à d'autres moments, ce sont les critères de qualité scolaire et psychotechnique qui sont mis en avant pour le recrutement. Dans l'ensemble, la proportion des enfants de salariés Peugeot reste élevée, et relativement stable. L'école fonctionne comme vecteur de promotion sociale pour une 
partie des familles de salariés. Une fois entrés dans l'usine, la plupart de ses anciens élèves connaissent une belle carrière, finissant souvent parmi la maîtrise, les techniciens et l'encadrement. Porteurs d'un fort esprit de corps, traducteurs et adaptateurs des volontés managériales tout en connaissant de l'intérieur le monde des ateliers, ils constituent un réseau central dans le monde sochalien dont ils forment de fait l'ossature sociale. Mais la création des lycées techniques change la donne. Celui de Montbéliard, ouvert en 1960, forme un pôle d'attraction considéré comme porteur de plus d'avenir. En une décennie, l'École d'apprentissage Peugeot s'affaiblit, et cesse de recruter en 1970. L'apprentissage d'entreprise a laissé la place à l'enseignement technique assuré par l'Éducation nationale.

13 C'est une autre dynamique qui marque la vente des voitures à tarif préférentiel au personnel, encore peu étudiée. Au cours des années cinquante, l'achat d'automobile se diffuse dans le personnel comme au sein de la société. En 1962, un salarié sur six achète une voiture neuve dans l'année ${ }^{18}$. Rapidement, les salariés se font revendeurs, si bien qu'ils deviennent le premier réseau de vente de la marque. Trouvant leurs clients dans leur entourage ou par petite annonce, ils se font promoteurs de leur production, représentants de l'entreprise. Cette activité lucrative les rend sensibles aux variations de conjoncture ainsi qu'à la gamme de modèles proposée.

La chronologie proprement industrielle affecte plus directement les politiques de logement et de transport du personnel. Peugeot s'engage en effet après la Reconstruction, dans d'énormes efforts visant à mettre des effectifs suffisants au service de la croissance industrielle de son usine.

Le déploiement d'un réseau de transports dans la région est un élément indispensable de la mobilisation de main-d'œuvre dans le bassin régional. Pour cela, le train encore en vigueur après la Seconde Guerre mondiale est remplacé par des camions puis, rapidement, par des cars de ramassage qui s'enfoncent à des dizaines de kilomètres à la ronde. L'essor de l'usine automobile profite des concentrations mises en œuvre dans l'agriculture aussi bien que de l'essoufflement de la vieille métallurgie et de l'industrie textile. Au cours des années soixante, l'essentiel de la conquête de cette main-d'œuvre régionale est effectué, au prix coûteux de centaines de cars effectuant leur tournée quotidienne. L'énorme réseau, le premier de France pour une entreprise privée, subit des compressions, à l'occasion de réductions d'effectifs qui interviennent au cours des années quatre-vingt et quatre-vingt-dix. Certaines suppressions de lignes de ramassage contribuent à rendre plus difficile et plus coûteux pour les salariés éloignés la conservation de leur emploi chez Peugeot.

16 Parallèlement, Peugeot entreprend de regrouper à proximité le plus de personnel possible. Outre les cités qui portent son nom, et datent de la première moitié du siècle, il lui faut faire feu de tout bois. Des foyers sont construits, dans lesquels des centaines puis des milliers de salariés, mariés ou non, vivent en célibataires. Mais les lits ne suffisent pas et l'inconfort fait repartir une partie de cette main-d'œuvre. L'entreprise encourage et soutient financièrement les mouvement de Castors par lequel des ouvriers s'entraident à construire leur maison. Surtout, elle participe à un organisme local de HLM qui s'engage dans de grands programmes de construction. Dans plusieurs communes avoisinantes sont construits de grands ensembles de plusieurs milliers de logements dont la plupart sont attribués à des salariés de Peugeot ${ }^{19}$. Rapporté aux économies réalisées en matière de transport, le coût des investissements est amorti à échéance moyenne, outre l'avantage de ne plus être à la merci des aléas climatiques pour le ramassage du personnel. Aux côtés 
des immeubles, viennent s'adjoindre des quartiers de pavillons où s'installent, en propriétaires, des cadres laissant les «blocs » à des ouvriers venant s'installer en famille. Pour certains salariés, des dispositions dessinent une trajectoire résidentielle, de la vie en foyer coïncidant avec les derniers temps de leur domiciliation à la campagne ou dans d'autres régions, vers l'ancrage dans l'agglomération par l'appartement HLM puis l'acquisition du pavillon. Chaque stade de cette progression renouvelle le lien avec l'entreprise, par les fidélités tissées avec le service logement ou, plus lourdement, les emprunts contractés. À côté de cet idéal-type, les options et opportunités personnelles occasionnent une grande variété de situations, parfois totalement autonomes de l'entreprise, parfois aussi recomposées par celle-ci. Certains cadres s'associent ainsi, au cours des années soixante, pour acquérir des terrains et y faire bâtir leur maison. L'entreprise structure longtemps la spécificité résidentielle de ses travailleurs coloniaux ou étrangers. Certains foyers des années cinquante sont nommés Douars afin de paraître plus accueillants à la main-d'œuvre venant d'Algérie. Plus tard, une association autonome construit d'autres foyers pour travailleurs étrangers. Puis les regroupements familiaux s'effectuent dans plusieurs cités que quittent les candidats au logement pavillonnaire. Peugeot, au demeurant, réexamine sa politique au vu des péripéties qui marquent une grève survenant en 1965. Dans ce conflit, qui porte essentiellement sur des revendications salariales, la concentration récente de salariés dans les grands ensembles en cours de construction alimente un vivier de solidarité ouvrière et favorise la formation d'une identité collective auprès des groupes de nouveaux salariés venus des milieux ruraux de la région ${ }^{20}$. Pour l'entreprise, l'alerte entraîne un réajustement stratégique. L'option de rapprocher son personnel perd son attrait, tandis que le profil des ouvriers-paysans retrouve du charme. Ce type de salarié, plutôt mal considéré jusque-là en raison du faible attachement à l'entreprise, devient plus rassurant du fait même de son autonomie. Au cours des années soixante-dix, les programmes d'ensembles locatifs sont ralentis, au profit des aides à l'accession à la propriété. Le début des années quatre-vingt est marqué par des réductions multiformes d'effectifs, notamment parmi les travailleurs étrangers. Certaines cités HLM se dégarnissent, quelques cages d'escalier sont désaffectées. En matière immobilière, l'entreprise accentue son désengagement.

\section{Du côté du travail : un resserrement des marges techniques}

17 En matière d'organisation du travail, les grands principes sont en place depuis l'entredeux guerres. Les activités qui constituent le cœur de la fabrication sont concentrées spatialement, et leur disposition suit un souci de circulation des pièces correspondant au processus de production. L'orchestration d'ensemble est assurée par l'encadrement, qui se dote de services fonctionnels tels que les études du produit, les méthodes de fabrication ou le contrôle de la qualité. Cet encadrement établit son emprise sur le monde des ateliers, c'est-à-dire sur les ouvriers mais aussi, et l'enjeu ne doit pas être sous-estimé, sur la maîtrise. Cette dynamique, souvent réduite aux seuls principes du taylorisme, prend en réalité des formes particulières ${ }^{21}$. Surtout, l'histoire des pratiques fait ressortir des changements significatifs dans les conditions d'exercice du travail et des relations qui s'y nouent.

18 Tout d'abord, le temps du travail est recomposé, sous l'effet d'une tendance à diffuser l'activité en continu des installations, et des hommes qui les servent. Les horaires de 
l'usine s'allongent, en deux vagues. La première consiste en une extension des horaires de doublage, c'est-à-dire d'une production réalisée en deux équipes, deux horaires qui se succèdent. Cette évolution s'effectue dans les années cinquante-soixante, et place l'essentiel du personnel de fabrication dans des horaires allant de $4 \mathrm{~h}$ à $13 \mathrm{~h}$ une semaine, et de $13 \mathrm{~h}$ à $22 \mathrm{~h}$ la semaine suivante. Au cours des années quatre-vingt intervient une deuxième vague d'extension, avec la création d'une équipe de nuit, pour le même personnel de fabrication, ou du travail de week-end. Ces deux vagues se retrouvent sous des formes voisines et des calendriers proches dans la plupart des autres usines automobiles, signe d'une évolution de fond dans l'emploi de la main-d'œuvre. Les conséquences de ces changements sont aussi considérables que sous-estimées, tant l'attention se focalise sur la durée du travail. De tels rythmes bousculent, certes, les rythmes biologiques d'autant plus qu'aux horaires de travail proprement dits s'ajoutent ceux des transports. Ils ont des dimensions sociales importantes, à commencer par l'intimité familiale qui se trouve structurée de façon tyrannique par l'absence du salarié concerné ou par sa présence envahissante à travers ses contraintes de sommeil à des moments atypiques. L'alternance que pratiquent parfois des conjoints pour des raisons de frais de garde mettent à rude épreuve les relations de couple. L'emprise de l'usine s'impose aussi dans la vie associative, de voisinage, et établissent des clivages étanches entre réseaux définis par leurs horaires à l'usine.

Seconde tendance de longue durée, l'extension du principe de la chaîne. Sa mise en place, au cours des années vingt, est très restreinte et ce principe n'est appliqué que peu à peu à des travaux de moins en moins rudimentaires. La chaîne conquiert progressivement les fabrications de fonderie, de forge puis, après la Seconde Guerre mondiale, d'usinage des pièces mécaniques. L'assemblage d'ensembles mécaniques, puis de moteurs, servent de préludes à l'assemblage en chaîne de carrosseries, puis des voitures complètes. Chacune de ces progressions signifie le progrès du mouvement imposé au détriment de l'autonomie rythmique des ouvriers ${ }^{22}$. Puis, dans les années cinquante, la contrainte enchaînée change d'envergure : elle enchaîne les ateliers entre eux par l'automatisation du transport des pièces de l'un à l'autre, par des convoyeurs mécaniques qui traversent les murs et les allées. Progressivement, l'unicité du mouvement tend à s'étendre à l'échelle du site de Sochaux. Mais des difficultés de coordination entravent cette mise au pas généralisée des activités, jusqu'à ce que l'intégration des bases de données informatiques et des logiciels de gestion des flux, des stocks et des travaux permette, au cours des années quatre-vingt, de resserrer le contrôle de l'activité globale. Les pannes et autres aléas, sources d'autant d'entrebaillements dans la rigueur usinière, sont réduits en même temps que les marges de précaution en temps, en ressources et en personnel, que prenaient à tous les niveaux les responsables pour tenir leurs engagements.

20 La base de la mise sous contrôle du travail ouvrier réside, comme on l'attend, dans l'affinement incessant de la décomposition du travail ouvrier. Le principe a pris forme au début $d u x^{*}$ siècle, avec l'apparition du chronométrage et la référence explicite à Taylor dans l'entreprise Renault en 1907 puis $1913^{23}$. Là aussi, le suivi des pratiques permet à l'histoire de suivre les changements qui affectent les situations effectives de travail. En la matière, les pratiques d'organisation sont à relier aux traces du travail des salariés qui en sont la cible ainsi qu'aux modalités de rémunération, qui traduisent un état de l'organisation effective du travail ouvrier ${ }^{24}$. À l'époque de la reconstruction, l'entreprise met en œuvre la méthode Bedaux, une des méthodes de gestion et d'organisation du travail inspirées du taylorisme ${ }^{25}$. Cette méthode s'appuie sur le salaire au rendement (ou 
au «boni»), qui établit, grâce à la mesure des techniciens, des normes de travail de référence et qui incite à dépasser ces normes grâce à une bonification de salaire. Un tel système, qui reprend les préconisations de Taylor, est aussi un aveu de relative faiblesse des organisateurs. Ces derniers, en effet, s'en remettent aux intéressés pour fixer leur propre charge de travail, eux-mêmes ne pouvant faire mieux que d'entériner périodiquement les niveaux atteints en les convertissant en nouvelle norme de référence. L'organisation scientifique est encore fondée sur l'arbitrage qu'effectuent les ouvriers entre leur surcroit d'effort et leur attente de gain additionnel. Au cours des années cinquante, Peugeot fournit un énorme effort de mesure. Les techniciens de services de méthode et de chronométrage, dont le nombre est considérablement accru, s'engagent dans un vaste travail d'analyse des postes de travail et des opérations, sous le vocable de simplification du travail. Mois après mois, les rapports du directeur font état des centaines de chronométrages effectués, balisant l'énorme travail fourni par les services techniques pour disséquer les opérations en gestes et pour fabriquer des temps correspondants. Le résultat de cette vague rationalisatrice est illustré par la suppression du salaire au rendement, par ailleurs décrié par les syndicats. Affirmant sa prétention à pouvoir fixer en toute rationalité l'effort exigible, l'entreprise supprime l'effort variable et instaure un salaire fixe dont le taux ne dépend plus que des particularités du poste occupé. Après 1968, l'organisation est contestée de plusieurs façons. L'émiettement, la répétition et l'ennui accentués par cette vague de rationalisation suscitent le surcroît de désaffection ouvrière évoqué plus haut. Les réponses apportées consistent en des expériences alternatives d'organisation regroupées sous la formule d'amélioration des conditions de travail. Entre autres résultats, ce réexamen du travail, associé à la poursuite de la rationalisation, facilite l'effacement du salaire au poste et la simplification des classifications, au profit d'une individualisation plus affirmée.

À travers les séquences successives ainsi évoquées, plusieurs tendances se complètent sur la longue durée. Hormis le travail sur machine, souvent réduit à des gestes élémentaires depuis l'époque de Simone Weil ${ }^{26}$, l'activité ouvrière est de plus en plus fragmentée. Ainsi sur les chaînes, le temps correspondant à un cycle d'opérations passe de quatre ou six minutes à la fin des années quarante à une minute cinquante ans plus tard. La décomposition gestuelle correspondante s'accompagne d'un transfert. Ces gestes deviennent de plus en plus simples et abstraits tandis qu'ils s'appliquent à des outils, voire à des installations de plus en plus complexes. Les équipements de travail s'enrichissent (en même temps que la voiture elle-même et ses composants). Dans le travail, l'interaction se resserre en direction de l'objet lui-même tandis qu'elle se fait de plus en plus complexe avec le système de fabrication, à travers la multiplication des informations échangées avec les machines et autres lecteurs, les collègues, la hiérarchie ou les différents services fonctionnels. Cette interdépendance accentuée des salariés dans le dispositif renouvelle, en quelque sorte, les considérations de Marx selon lesquelles le capital fixe se renforce au détriment du capital variable dans l'évolution de la production industrielle.

\section{Conclusion}

Pour plus de commodité, l'analyse a distingué deux aspects dans la relation de travail, l'emploi et l'activité proprement dite. La coupure est forcément problématique, tant les partenaires, employeurs comme salariés, mettent constamment en rapport ces deux 
références. Elle correspond toutefois à des catégories de la vie ordinaire. Ainsi, couramment, des ouvriers apprécient l'emploi, la «place » chez Peugeot dont ils louent les atouts, tandis qu'ils déplorent le travail qu'ils y exécutent. Du côté du poste, l'activité est de plus en plus densément prise dans un réseau d'interdépendances techniques tandis que se réduit le face-à-face avec l'objet du travail, l'autorité du contremaître tend à se faire relayer par celle des fiches de suivi et autres écrans de contrôle ${ }^{27}$. Plus nettement qu'une accélération des cadences dont les repères s'avèrent fuyants ${ }^{28}$, c'est cette densification des modalités de l'activité qui marque l'évolution. Toutefois l'activité reste caractérisée par l'interdépendance plutôt que par une dépendance stricto sensu, l'interaction reste présente au cœur de la prescription. Côté place, le marchandage de l'emploi est certes inégal entre employeur et salarié. Mais les variations d'équilibre entre l'offre patronale et le consentement ouvrier rappellent que la relation reste constamment sujette à la défection salariée ${ }^{29}$ comme au retrait de l'entreprise. La tendance de l'usine à modeler sa sphère d'influence doit s'accommoder de la conjoncture industrielle et du contexte institutionnel, dont les salariés tiennent compte eux aussi. D'une part, les dispositions prises par Peugeot s'inscrivent tout d'abord dans un paysage politique, institutionnel économique qui limitent les marges d'action de l'entreprise et pèsent sur ses choix. D'autre part, les variations de perspectives conduisent les acteurs à réévaluer le sens des dispositifs sociaux mis en place. De façon générale, les années cinquante et soixante voient l'entreprise effectuer ou maintenir des dispositifs sociaux dans toutes les directions tandis que les décennies quatre-vingt et quatre-vingt-dix sont marquées par un repli général. Ainsi, la croissance forte, qui s'accompagne d'importants besoins, impliquent ces efforts afin d'attirer et de garder le personnel nécessaire et contrecarrer les différentes formes de dérobade («turn-over», absentéisme etc.) relevées dans d'autres lieux ${ }^{30}$. Peugeot met alors en œuvre, pour reprendre la formule d'un ancien responsable de ressources humaines, une «technologie sociale $»^{31}$ adaptable. Puis, sous la pression du chômage, l'emploi reprend sa force d'attraction. Il permet d'alléger le dispositif et de resserrer en même temps la proportion des bénéficiaires en externalisant des activités et en systématisant le recours aux intérimaires. L'entreprise se voit alors rappeler ses responsabilités sociales par les partenaires sociaux et institutionnels. $\mathrm{Au}$ regard de ces grandes tendances, le système social de l'entreprise apparaît ainsi, non pas comme la construction d'une domination totale mais comme le lieu d'interaction inégales entre acteurs sociaux.

\section{NOTES}

1. De ces recompositions du lien de travail, vues sous l'angle de la subordination, Patrick Fridenson a dressé deux tableaux sur la longue période industrielle et pour des activités variées. Cf. Patrick Fridenson, "La subordination dans le travail. Les questions de l'historien ", in JeanPierre Chauchard et Anne-Chantal Hardy-Dubernet (dirs.), La subordination dans le travail. Analyse juridique et sociologique de l'évolution des formes d'autonomie et de contrôle dans la relation de travail, Paris, La Documentation française, 2003, p. 59-69; «Les transformations des pratiques de subordination dans les entreprises et l'évolution du tissu productif en France », in Héloïse Petit et 
Nadine Thèvenot (dir.), Les nouvelles frontières du travail subordonné. Approche pluridisciplinaire, Paris, La Découverte, 2006, p. 21-46.

2. André Gueslin, "Le paternalisme revisité en Europe occidentale (seconde moitié du XIXe, début XXe siècle)", Genèses, n7, mars 1992, p. 201-211. Dans son chapitre introductif au livre qu'elle édite sur le sujet, Sylvie Schweitzer ouvre la définition. Cf. Sylvie Schweitzer, " "Paternalismes" ou pratiques sociales?», in Sylvie Schweitzer (dir.), Logiques d'entreprises et politiques sociales des XIXe et XXe siècles, Lyon, Programme Rhône-Alpes de recherches en sciences humaines, 1993, p 5-18.

3. Simone et Jean Lacouture, En passant par la France, journal de voyage, Paris, Le Seuil, 1982, p. 60.

4. Jean-Louis Loubet, Automobiles Peugeot, une réussite industrielle 1945-1973, Paris, Economica, 1990, p. 232-241; Nicolas Hatzfeld, Les gens d'usine. 50 ans d'histoire à Peugeot-Sochaux, Paris, les éditions de l'Atelier, 2002, p. 217-219.

5. Journal d'Information Peugeot, $\mathrm{n}^{\circ} 4,16$ avril 1970.

6. Évelyne Desbois, Yves Jeanneau, Bruno Mattei, La foi des charbonniers. Les mineurs dans la bataille du charbon, 1945-1947, Paris, Éditions de la Maison des Sciences de l'Homme, 1986.

7. Gérard Noiriel, Les ouvriers dans la société française, Paris, Le Seuil, 1986, p.198-199.

8. En l'occurrence, les fédérations de la métallurgie de la CGC, de FO puis de la CFTC.

9. Nicolas Hatzfeld, "Les ouvriers de l'automobile: des vitrines sociales à la condition des OS, le changement des regards ", in Geneviève Dreyfus-Armand, Robert Franck, Marie-Françoise Lévy, Michelle Zancarini-Fournel (dirs.), Les années 68. Le temps de la contestation, Bruxelles, Éditions Complexe - IHTP/CNRS, 2000, p. 345-362.

10. Claude Poperen, Renault, regards de l'intérieur, Paris, Éditions sociales, 1983,p. 188-195 ; Laure Pitti, «Ouvriers algériens à Renault-Billancourt, de la guerre d'Algérie aux grèves d'OS des années 1970. Contribution à l'histoire sociale et politique des ouvriers étrangers en France ", thèse de doctorat d'histoire, université Paris-VIII, 2002.

11. Éric Pezet, « De la classification des emplois à la question des compétences. Modélisation des relations entre gestion des ressources humaines et négociation collective », thèse de doctorat en gestion, École nationale supérieure des mines de Paris, 2001.

12. En 1978, un document de travail de la direction du personnel et des relations sociales de Sochaux s'intitule "Objectifs: démassifier, revaloriser-hiérarchie, personnaliser ». Pour ses auteurs, il s'agit de sortir d'une gestion dite taylorienne ou de masse, du personnel ouvrier. $\mathrm{Cf}$. Nicolas Hatzfeld, Les gens d'usine [...], op. cit., p. 385-389 (supra note 4).

13. Stéphane Beaud et Michel Pialoux, Retour sur la condition ouvrière. Enquête aux usines Peugeot de Sochaux-Montbéliard, Paris, Fayard, 1999.

14. Jean-Paul Goux, Mémoires de l'enclave, Arles, Actes sud, 2003 (Paris, Mazarine, 1986).

15. Yves Cohen, «L'invention des techniciens sociaux. Du commandement social après juin 1936 chez Peugeot ", Actes de la recherche en sciences sociales, septembre 1996, p. 30-43.

16. Nicolas Hatzfeld, Les gens d'usine [...], op. cit., p. 202-206 (supra n. 4).

17. Une première école a été créée en 1919 par Peugeot dans la ville voisine de Beaulieu, berceau des fabrications automobiles et de cycles. Cf. Nicolas Hatzfeld, «l'École d'Apprentissage Peugeot

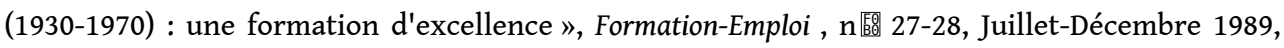
p. $115-130$.

18. Nicolas Hatzfeld, Les gens d'usine [...], op. cit., p. 206-208.

19. Stéphane Jonas, "Mémoire et quartiers populaires sensibles: étude de cas de l'“archipel Peugeot" de la région industrielle Montbéliard-Mulhouse ", in Liane Mozère, Michel Peraldi et Henri Rey (dir.), Intelligence des banlieues, La Tour d'Aigues, Éditions de l'aube, 1999, p. 110-120.

20. Bernard Dézert, «Quelques aspects géographiques de la grève des usines de la S.A. Peugeot en avril-juin 1965 ", Bulletin de l'Association des Géographes français, n㬝 340-341, janvier-février 1966, p. 20-36. 
21. Yves Cohen, Organiser à l'aube du taylorisme. La pratique d'Ernest Mattern chez Peugeot, 1906-1919, Presses Universitaires de Franche-Comté, Besançon, 2001.

22. Sylvie Schweitzer, Des engrenages à la chaîne. Les usines Citroën 1915-1935, Lyon, PUL, 1982 ; Alain Michel, «Les images du travail à la chaîne dans les usines Renault de Boulogne-Billancourt (1917-1939). Une analyse des sources visuelles : cinéma, photographies, plans d'implantation », thèse d'histoire, École des Hautes Études en Sciences Sociales, 2001.

23. Patrick Fridenson, « Un tournant taylorien de la société française (1904-1918) », Annales E.S.C. , n嘼 5, septembre-octobre 1987, p. 1031-1060.

24. Bernard Mottez, Systèmes de salaire et politiques patronales. Essai sur l'évolution des pratiques et des idéologies patronales, Paris, éditions du CNRS, 1966; William Grossin, Le travail et le temps: horaires, durée, rythmes, Paris, Anthropos, 1969.

25. Aimée Moutet, Les logiques de l'entreprise. La rationalisation dans l'industrie française de l'entredeux-guerres, Paris, éditions de l'EHESS, 1997 ; Eric Geerkens, La rationalisation dans l'industrie belge de l'entre-deux-guerres, Bruxelles, Palais des Académies, 2004.

26. Simone Weil, Écrits historiques et politiques, (t. II), L'expérience ouvrière et l'adieu à la révolution (juillet 1934-juin 1937), Paris, Gallimard, 1988.

27. Nicolas Hatzfeld, « De l'arme patronale au recours ouvrier, la mutation du chronomètre », in Dominique Barjot (dir.), Le travail à l'époque contemporaine, Paris, éditions du CTHS, 2005, p. 127-141.

28. Nicolas Hatzfeld, «L'intensification du travail en débat. Ethnographie et histoire aux chaînes de Peugeot-Sochaux », Sociologie du travail, n 46, 2004, p. 291-307.

29. Albert Hirschman, Défection et prise de parole : théorie et applications, Paris, Fayard, 1995 (1 ère édition 1972).

30. Sylvie Schweitzer (dir.), Logiques d'entreprises [...], op. cit. ; Gérard Noiriel, « Du "patronage ” au "paternalisme" : la restructuration des formes de domination de la main-d'œuvre ouvrière dans l'industrie métallurgique française ", Le Mouvement Social, $\mathrm{n}^{\circ} 144$, juillet-septembre 1988, p. 35 ; Piero Galloro, Ouvriers du fer, princes du vent. Histoire des flux de main-d'œuvre dans la sidérurgie lorraine, Metz, éditions Serpenoise, 2001.

31. François Cusey, entretien avec Alain Kopff, 4 janvier 1999. Ce responsable emploie l'idée de technique sociale dans un sens très proche de celui que lui donne Yves Cohen dans «L'invention des techniciens sociaux [...]», art. cit., p. $30-43$ (supra note 15).

\section{AUTEUR}

\section{NICOLAS HATZFELD}

Université d'Évry 